\title{
Adaptive Gain PID Control for Mechanical Systems
}

\author{
Ricardo Guerra, Salvador González and Roberto Reyes \\ Universidad Autónoma de Baja California \\ México
}

\section{Introduction}

The design and use of PID controllers is a part of what has been denominated Classical Control, which as the name implies, has been studied for many years (DiStefano et al. 1996), however it continues to be a source for research (Alvarez et al. 2008), (Ang et al. 2008), (Su et al. 2010).

The structure of the controller contains a differential term to aid in the reduction of system friction and an integral term to attenuate steady state error. The drawbacks of this control scheme, particularly for nonlinear mechanical systems, include the difficulty in selecting adecuate controller gains, a process usually refered to as tuning. The difficulty usually lies in the fact that if the controller gains are set too small, the control objective may never be reached, whereas the selection of excesively large controller gains may result in system instability. Many approaches have been proposed to properly tune PID gains (Ang et al. 2008), (Chang \& Jung 2009), (Su et al. 2010), others have tried to improve upon the performance of the PID controller by including modern control techniques such as neural networks, fuzzy logic or variable structure control (Guerra et al. 2005).

Among these, variable structure control, specifically sliding mode control, has shown to possess certain desirable properties, such as disturbance rejection and finite time convergence; however it also presents unwanted behaviors mainly high frequency switching, a phenomenon refered to as chattering, which is undesirable in mechanical systems because it can cause accelerated wear of the mechanical components as well as activate unmodeled dynamics. One solution presented is to include an adaptive gain in the high frequency term so that the desirable properties may be exploited, and the undesirable effects minimized, achieving an enhanced performance (Guerra et al. 2005).

\section{Background}

The control of mechanical systems is subject to many difficulties, as evidenced by the research devoted to such aspects of mechanical systems as dead zone (Zhang \& Gen 2009), and friction (Canudas de Wit et al. 1995).

Consider a first order mechanical system given by (Canudas de Wit et al. 1995) 


$$
\ddot{x}=\frac{u-f(\dot{x})}{m}
$$

where $x$ is the position variable, $m$ is the mass, $u$ is the control input and the function $f(\dot{x})$ denotes the nonlinear friction force. The PID control law is given by (Canudas de Wit et al. 1995):

$$
u=K_{p} e+K_{i} \int_{0}^{t} e(\tau) d \tau+K_{d} \dot{e}
$$

where $K_{p}, K_{d}$ and $K_{i}$, are the proportional, derivative and integral gains, respectively and the error term is given by $e=x-x_{d}$, where $x_{d}$ is the constant desired value.

Mechanical systems under integral control action have been known to present limit cycles, due in part to the complex nature of the friction force. This results in the system never reaching the desired position (Canudas de Wit et al. 1995).

The authors in (Guerra et al. 2005) present an approach considering a PD controller which is modified by the inclusion of a neural networks chattering controller that allows the high frequency swithching when the system is away from the desired position, but tends to vanish once the desired position is reached. In this chapter we will build upon that result and apply a similar stragegy to a PID controller.

\section{Controller design}

Consider the system (1) with unit mass and friction force given by (Makkar et al. 2005):

$$
f(\dot{x})=\gamma_{1}\left[\tanh \left(\gamma_{2} \dot{x}\right)-\tanh \left(\gamma_{3} \dot{x}\right)\right]+\gamma_{4} \tanh \left(\gamma_{5} \dot{x}\right)+\gamma_{6} \dot{x}
$$

The objective is for the error $e$ to reach zero, i.e.,:

$$
\lim _{t \rightarrow \infty} e(t)=0
$$

where

$$
e=x-x_{d}
$$

to achieve this, the controller (2) is modified to:

$$
u=-K_{p} e-K_{i} \zeta-\left[2 \varepsilon+\delta K_{d}\right] \dot{e}
$$

where

$$
\begin{gathered}
\dot{\zeta}=e \\
\dot{\delta}=-\alpha \ln (\delta+1)+K_{r} \frac{[\delta+1]}{\ln (\delta+1)+1} e^{2}
\end{gathered}
$$

where $\varepsilon>0, \alpha>0$ and $K_{r}>0$ are constant parameters. The term $\zeta$ is used for simplicity in place of the term $\int_{0}^{t} e(\tau) d \tau$. It should be noted that for an intnitial condition $\delta\left(t_{0}\right)=\delta_{0} \geq 0$, $\delta(t) \geq 0$, for all $t \geq t_{0}$ (Hench, 1999). In addtion, the adaptive gain can be considered to be bound by $\delta \leq \delta_{M}$ by taking into account that a practical controller is subject to saturation. 


\section{Closed loop system}

To analyze the stability of the closed loop system, the following variable change is introduced:

$$
\omega=\varepsilon \zeta+e
$$

which is used to form the vector $\xi=\left[\begin{array}{lll}\omega & e & e\end{array}\right]^{T}$. Using equations (1), (3), (5), (6) and (7) the dynamic of the closed loop system is given by:

$$
\dot{\xi}=A(\delta) \xi+B(\dot{e})
$$

where

$$
\begin{gathered}
A(\delta)=\left[\begin{array}{ccc}
0 & \varepsilon & 1 \\
0 & 0 & 1 \\
-\varepsilon^{-1} K_{i} & -\left[K_{p}-\varepsilon^{-1} K_{i}\right] & -\left[2 \varepsilon+\delta K_{d}\right]
\end{array}\right] \\
B(\dot{e})=\left[\begin{array}{lll}
0 & 0 & -f(\dot{e})]^{T}
\end{array}\right.
\end{gathered}
$$

The state $\delta$ contained in $A(\delta)_{3,3}$ is governed by the dynamic adaptation law (8). By setting (8) and (10) to zero, it can be seen that the origin of the state space $(\xi=0, \delta=0)$ is the unique equillibrium for the system which, when applied to equation (9), implies $\zeta=0$.

\section{Stability analysis}

Consider the candidate Lyapunov function:

$$
V(\xi, \delta)=\xi^{T} P_{c} \xi+(\delta+1) \ln (\delta+1)
$$

where

$$
\begin{gathered}
P_{c}=\frac{1}{2}\left(P+P^{T}\right) \\
P=\left[\begin{array}{ccc}
\beta \varepsilon^{-1} K_{i} & 0 & 0 \\
0 & \beta\left[K_{p}-\varepsilon^{-1} K_{i}\right] & 0 \\
0 & 2 \beta \varepsilon & \beta
\end{array}\right]
\end{gathered}
$$

It should be noted that $V>0$ implies that $P_{c}>0$, which by applying Sylvester's Theorem (Kelly et al. 2005) requires that $\beta>0$, the complete analysis to ensure positivity of matrix $P_{c}$ is presented in the next section. To simplify stability analysis, the equality $\xi^{T} P_{c} \xi=\xi^{T} P \xi$ is considered so that expression (13) can be restated as

$$
V(\xi, \delta)=\xi^{T} P \xi+(\delta+1) \ln (\delta+1)
$$

The time derivative of (16) along the closed loop system (8) and (10) yields:

$$
\dot{V}=-\xi^{T} Q(\delta) \xi-R(\xi)-\alpha \ln (\delta+1)[\ln (\delta+1)+1]
$$

where

$$
R(\xi)=-B(\dot{e})^{T} P \xi-\xi^{T} P B(\dot{e})=2 \beta \varepsilon e f(\dot{e})+2 \beta \dot{e} f(\dot{e})
$$




$$
\begin{gathered}
W(\delta)=-\left[P A(\delta)+A(\delta)^{T} P\right] \\
Q(\delta)=\frac{1}{2}\left[W(\delta)+W(\delta)^{T}\right]-\hat{e}_{2} K_{r}(\delta+1) \hat{e}_{2}^{T} \\
=\left[\begin{array}{ccc}
0 & 0 & 0 \\
0 & 2 \beta \varepsilon\left[K_{p}-\varepsilon^{-1} K_{i}\right]-K_{r}(\delta+1) & \beta \varepsilon\left[2 \varepsilon+\delta K_{d}\right] \\
0 & \beta \varepsilon\left[2 \varepsilon+\delta K_{d}\right] & 2 \beta\left[\varepsilon+\delta K_{d}\right]
\end{array}\right]
\end{gathered}
$$

where $\hat{e}_{2}=\left[\begin{array}{lll}0 & 1 & 0\end{array}\right]^{T}$. Regarding equation (3) used in (18), every term in the expression can be bound by $b \tanh (c) \leq|b||c| \forall b, c \in \Re$. It can be stated that equation (3) satisfies:

$$
-e f(\dot{e}) \leq K_{\gamma}|e||\dot{e}|
$$

where

$$
K_{\gamma}=\gamma_{1}\left|\gamma_{2}-\gamma_{3}\right|+\gamma_{4} \gamma_{5}+\gamma_{6}
$$

it should be remembered that all the parameters $\gamma_{\iota}$ for $\iota=1 \ldots 6$ are positive constants. Regarding the term $\dot{e} f(\dot{e})$ in equation (18), it can be seen that this term is positive for $\gamma_{2} \geq \gamma_{3}>0$ by using the properties of hiperbolic functions in equation (3) and considering $\dot{e} \rightarrow \Theta \geq 0$ (first quadrant) we find that:

$$
\tanh \left(\left[\gamma_{2}-\gamma_{3}\right] \Theta\right)\left[1-\tanh \left(\gamma_{2} \Theta\right) \tanh \left(\gamma_{3} \Theta\right)\right] \geq 0
$$

given that $\Theta, \gamma_{2}$ and $\gamma_{3}$ are considered to be positive, the second term will always be non negative, whereas the first will be non negative if $\gamma_{2} \geq \gamma_{3}>0$ (as was previously stated). These considerations apply also when $\dot{e} \rightarrow \Theta \leq 0$ (third quadrant). By applying (21), (22) and (23) in (18), along with the previously stated $\delta \leq \delta_{M}$ equation (17) can be bounded by:

$$
\dot{V} \leq-\left[\begin{array}{l}
|e| \\
|\dot{e}|
\end{array}\right]^{T} Q_{c}\left[\begin{array}{l}
|e| \\
|\dot{e}|
\end{array}\right]-2 \beta \dot{e} f(\dot{e})-\alpha \ln (\delta+1)[\ln (\delta+1)+1]
$$

where

$$
Q_{c}=\left[\begin{array}{cc}
2 \beta \varepsilon\left(K_{p}-\varepsilon^{-1} K_{i}\right)-K_{r}\left(\delta_{M}+1\right) & \beta \varepsilon\left(2 \varepsilon+\delta_{M} K_{d}-K_{\gamma}\right) \\
\beta \varepsilon\left(2 \varepsilon+\delta_{M} K_{d}-K_{\gamma}\right) & 2 \beta \varepsilon
\end{array}\right]
$$

In the follwing section, a process for tuning the controller gains will be introduced, this will also be useful in provinding sufficient conditions to guarantee the positiviy of matrices $P_{c}$ and $Q_{c}$.

\section{Controller tuning}

In order to establish bounds on the controller gains, we first analyze the matrix $P_{c}$ defined in expression (14). To find the roots of this symmetric matrix, we apply Sylvester's Theorem (Kelly et al. 2005), which generates a cubic polynomial of the form $\varepsilon^{3}-3 b \varepsilon+2 a<0$ with $a=\frac{K_{i}}{2}$ and $b=\frac{K_{p}}{3}$ which is satisfied for $b^{3}>a^{2}$. Using $\exp (\bullet)$ to denote the exponential function, we define the terms $v_{1,2}=-a \pm i c=r \exp [\mp i(\theta-\pi)], c=\sqrt{b^{3}-a^{2}}, r=b^{\frac{3}{2}}$, $\theta=\arctan \left(\frac{c}{a}\right), \vartheta_{1,2}=2 a+v_{1,2}=r \exp ( \pm i \theta)$ and using Euler's formula the roots are: 


$$
\begin{gathered}
\varepsilon_{1}=-\left(p_{1}+p_{2}\right)=-2 r^{\frac{1}{3}} \cos \left(\frac{\theta}{3}\right) \\
\varepsilon_{2}=\sqrt{y_{1} y_{2}}=2 r^{\frac{1}{3}} \sin \left(\frac{\pi-2 \theta}{6}\right) \\
\varepsilon_{1}=q_{1}+q_{2}=2 r^{\frac{1}{3}} \cos \left(\frac{\pi-\theta}{3}\right)
\end{gathered}
$$

considering that $p_{\iota}=v_{\iota}^{\frac{1}{3}}, q_{\iota}=v_{\iota}^{\frac{1}{3}}$, and $y_{\iota}=p_{\iota}-q_{\iota}$ for $\iota=1,2$. Given that $c \in \Re^{+}, \theta \in\left(0, \frac{\pi}{2}\right]$. Taking then $\varepsilon_{1}<0$, and $0<\varepsilon_{2} \leq \varepsilon_{3}$. The polynomial $\varepsilon^{3}-3 b \varepsilon+2 a=\left(\varepsilon-\varepsilon_{1}\right)\left(\varepsilon-\varepsilon_{2}\right)\left(\varepsilon-\varepsilon_{3}\right)<0$ is satisfied for all $\varepsilon_{2}<\varepsilon<\varepsilon_{3}$. We propose the definition $b^{3}=\left(\sigma^{2}+1\right) a^{2}$ with $\sigma \gg 0$, in other words, the proportional gain in equation (6) is tuned as

$$
K_{p}=\left[\frac{27 K_{i}^{2}}{4}\left(\sigma^{2}+1\right)\right]^{\frac{1}{3}}
$$

Returning to $Q_{c}$ defined in expression (25), this matrix can be defined as positive by applying Sylvester's Theorem (Kelly et al. 2005) and tuning the derivitave gain in (6) as

$$
K_{d}=\frac{K_{\gamma}-2 \varepsilon}{\delta_{M}}
$$

the numerator in this equation must be positive, specifically, the constant bound from equation (22) must satisfy $K_{\gamma}>2 \varepsilon$, so from equations (26)-(30) the positivity of matrices $P_{c}$ and $Q_{c}$ is restricted to

$$
\max \left\{\frac{K_{i}}{K_{p}}, \frac{2 \beta K_{i}+K_{r}\left(\delta_{M}+1\right)}{2 \beta K_{p}}, \varepsilon_{2}\right\}<\varepsilon<\min \left\{\frac{K_{\gamma}}{2}, \varepsilon_{3}\right\}
$$

By establishing conditions to satisfy (31), which include the values of $K_{r}$ and $K_{i}$ selected to generate a valid range for $\varepsilon$, we can conclude that expression (13) is positive definite and that expression (17) is locally negative semi-definite, consequently the system (8) and (10) has a stable equilibrium at the origin. Moreover, by restricting $\eta=\left[\xi^{T} \delta\right]^{T}$ by the bounds $\eta_{\min } \leq \eta \leq \eta_{\max }$ and applying LaSalle's Principle (Kelly et al. 2005) to expression (24) a closed set can be defined as:

$$
\Omega=\left\{\eta \in \Re^{4}: \dot{V}(\eta)=0\right\}=\left\{\omega \in \Re,\left[\begin{array}{lll}
e & \dot{e} & \delta
\end{array}\right]^{T}=0\right\}
$$

Solving (32) along (8) and (10) it can be seen that

$$
\lim _{t \rightarrow \infty} \omega(t)=0
$$

and by invoking the variable change (9) that

$$
\lim _{t \rightarrow \infty} \zeta(t)=0
$$

therefore the origin of the system defined by (8) and (10) is locally asymptotically stable. 


\section{Simulation results}

In order to test the performance of the proposed controller simulations were carried out using the friction model (3) with the parameters set to $\gamma_{1}=1.25, \gamma_{2}=100, \gamma_{3}=10, \gamma_{4}=\gamma_{5}=1$., $\gamma_{6}=0.1, \alpha=10, \beta=1, \delta_{M}=1, \sigma=100, K_{i}=K_{r}=10$ and the mass is considered to be unitary.

Using the mentioned values in equations (22), (29) and (30) we obtain $K_{p}=188.9945$, $K_{\gamma}=122.6$ and applying the obtained values to equation (31) we arrive at $\max \{0.53,0.106,0.52\}<\varepsilon<\min \{61.3,13.7\}$ such that the value chosen was $\varepsilon=6.808$ and hence $K_{d}=108.9848$.

Figure 1 shows the performance of the position regulation. It should be noted that there is a very small overshot and that no limit cycles are present. The asymptotic stability can be easily seen in Figure 2 where the error is presented, it is clear that the error is still decreasing, achieving an accuracy within a micrometer after 200 seconds.

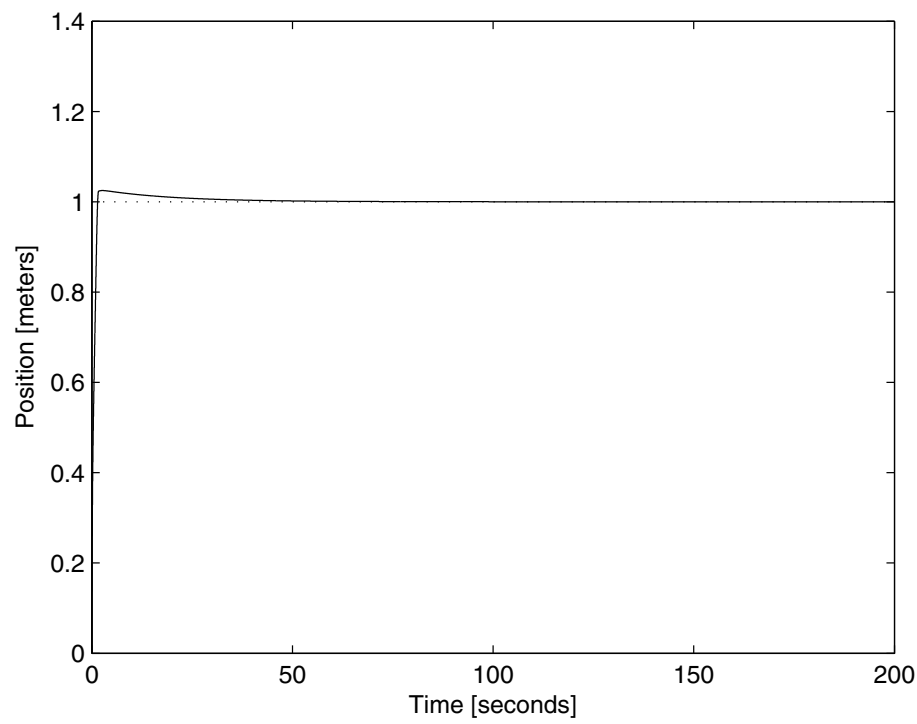

Fig. 1. Controller Performance: Achieved Position.

Figure 3 shows the evolution of the adaptive gain $\delta$, it is clear that as the error approaches zero, so too does the value of the adaptive gain, and consequently so does the value of the control variable, shown in Figure 4.

The control variable initially presents a large value which then decreases. It can be inferred from the asymptotic stability that the control variable decreses asymptotically with time as shown in Figures 4 and 5. Figure 5 shows the control variable in more detail. During the first ten seconds a small oscillation can be seen but it is eliminated after approximately 3 seconds. Figures 6 and 7 show that the term $\zeta=\int_{0}^{t} e(\tau) d \tau$ also approaches zero. It can be clearly seen, especially in Figure 7 that $\zeta$ asymptotically approaches zero. 


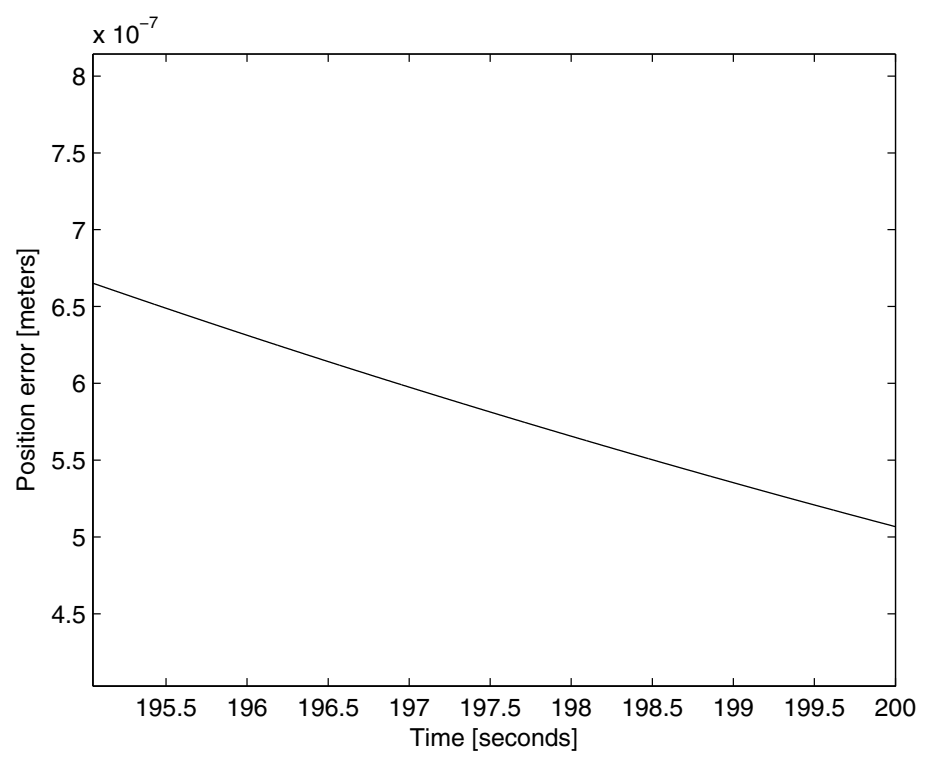

Fig. 2. Controller Performance: Position Error.

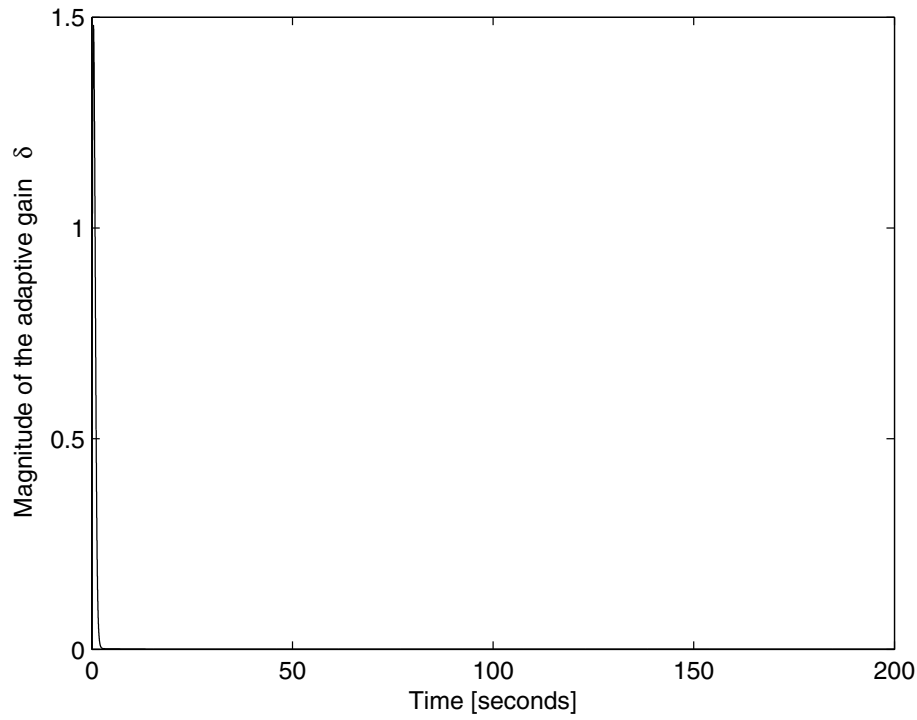

Fig. 3. Controller Performance: Adaptive Gain. 


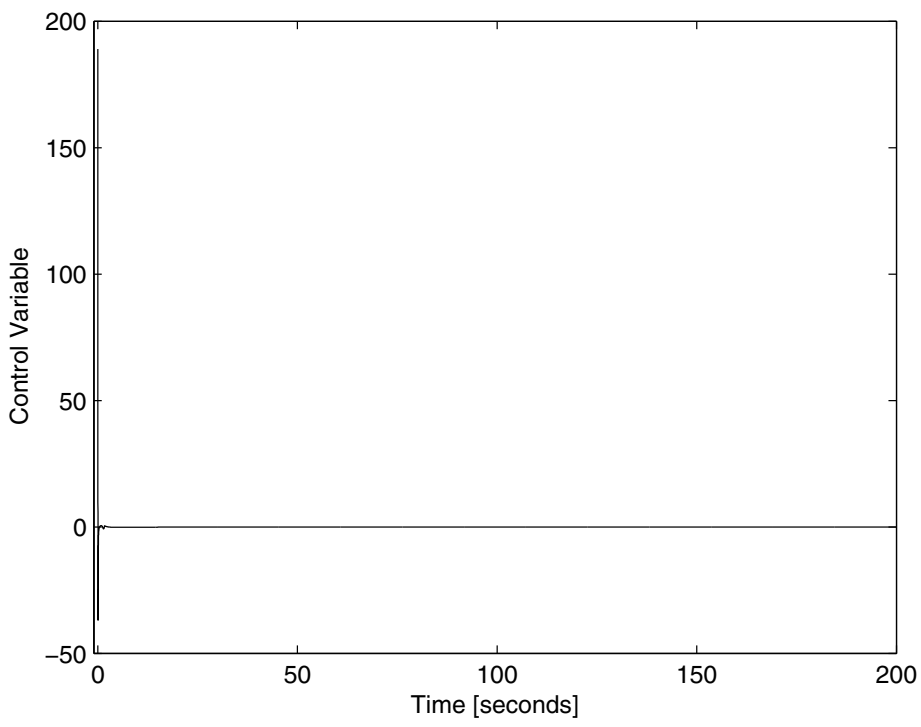

Fig. 4. Controller Performance: Control Variable.

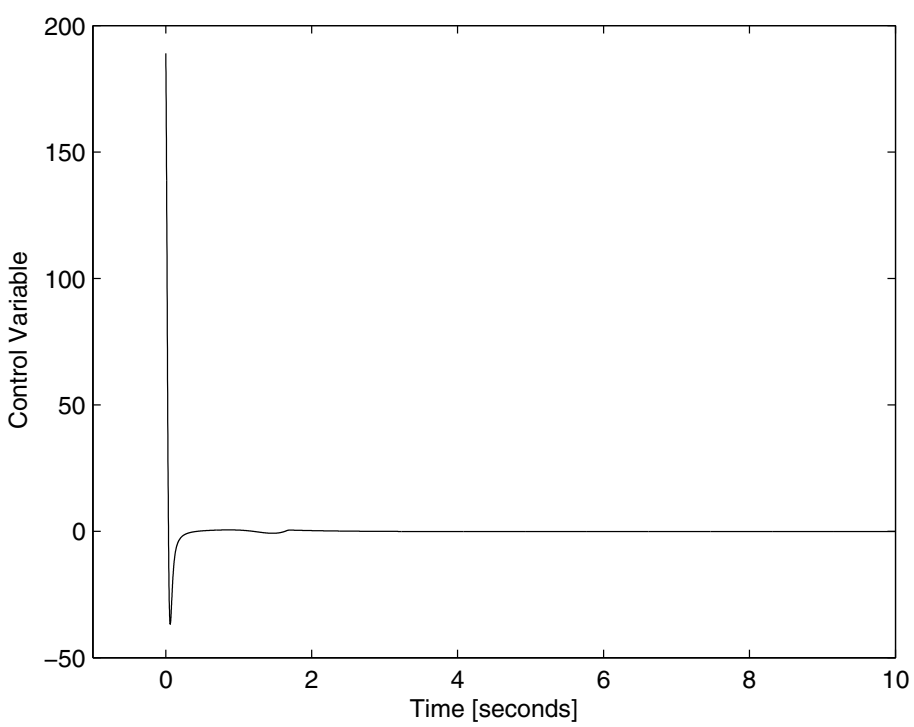

Fig. 5. Controller Performance: Control Variable (detail). 


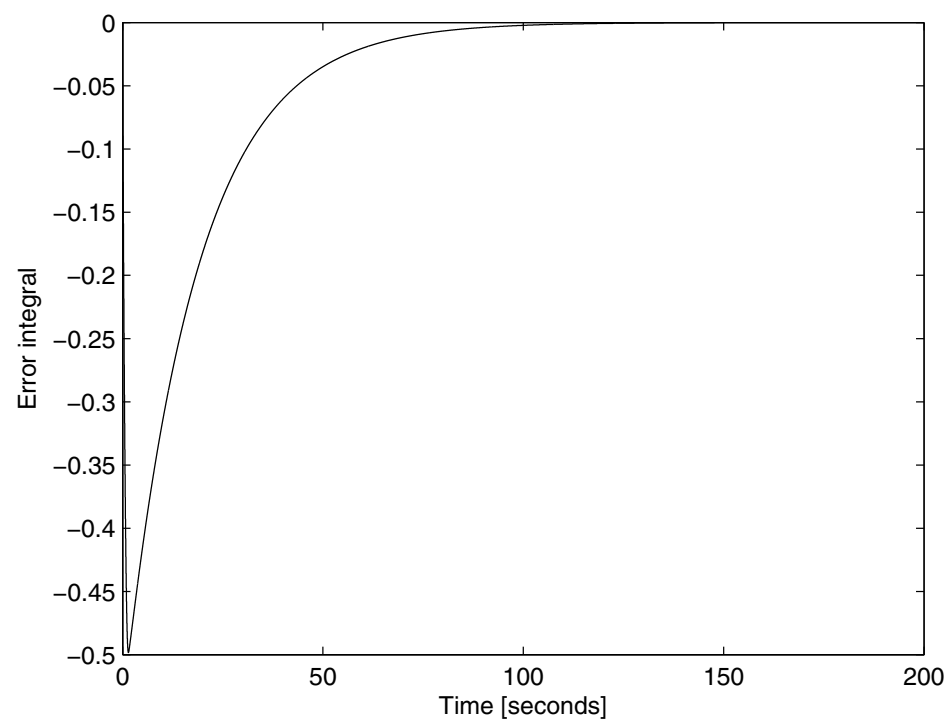

Fig. 6. Controller Performance: Error Integral.

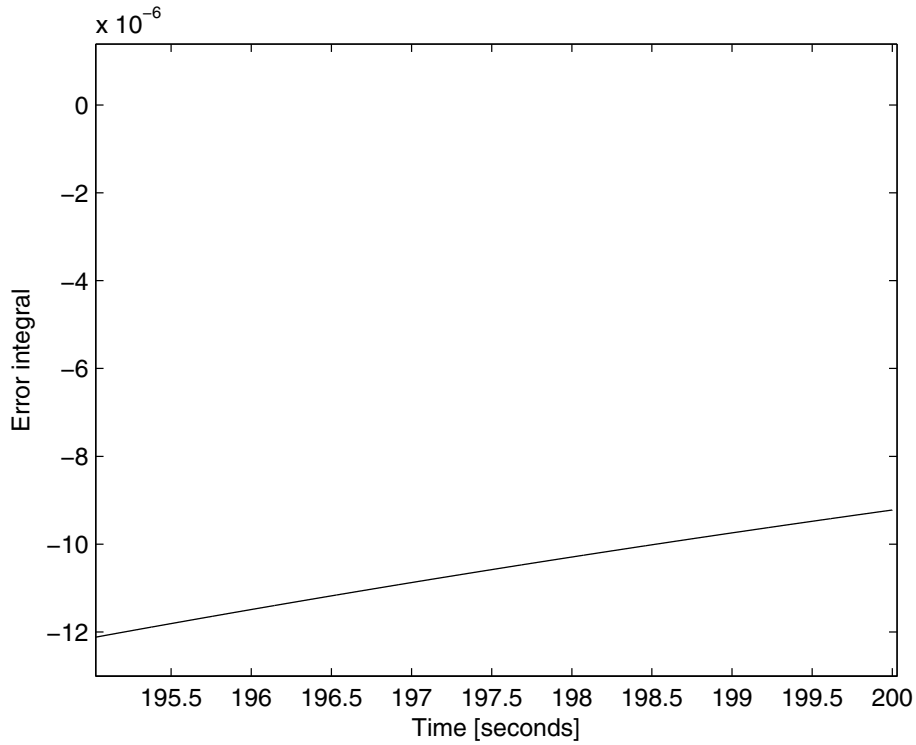

Fig. 7. Controller Performance: Error Integral (detail). 
Figure 8 shows how with increasing time, the value of the adaptive gain draws even closer to zero. The same can be said of the error in Figure 9 and of $\zeta$ in Figure 10.

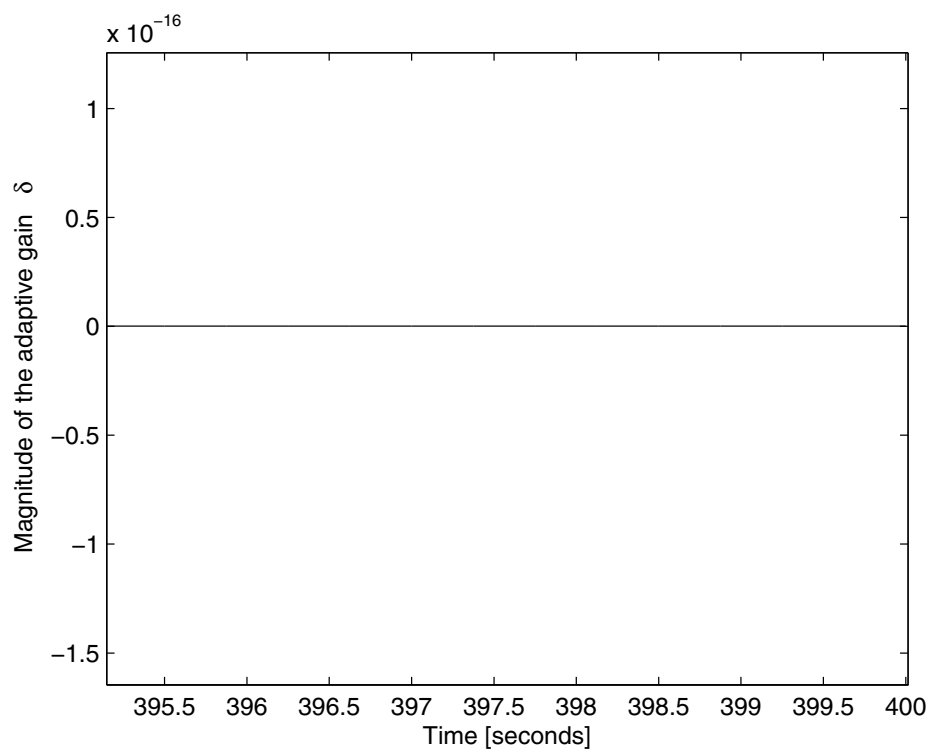

Fig. 8. Controller Performance: Adaptive Gain (detail).

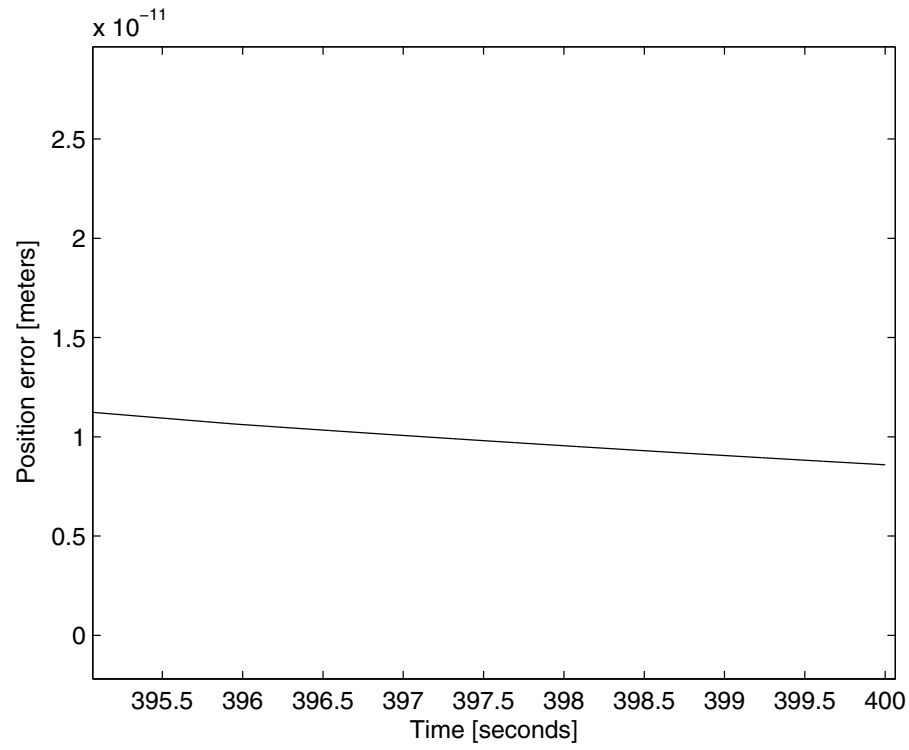

Fig. 9. Controller Performance: Position Error (detail). 


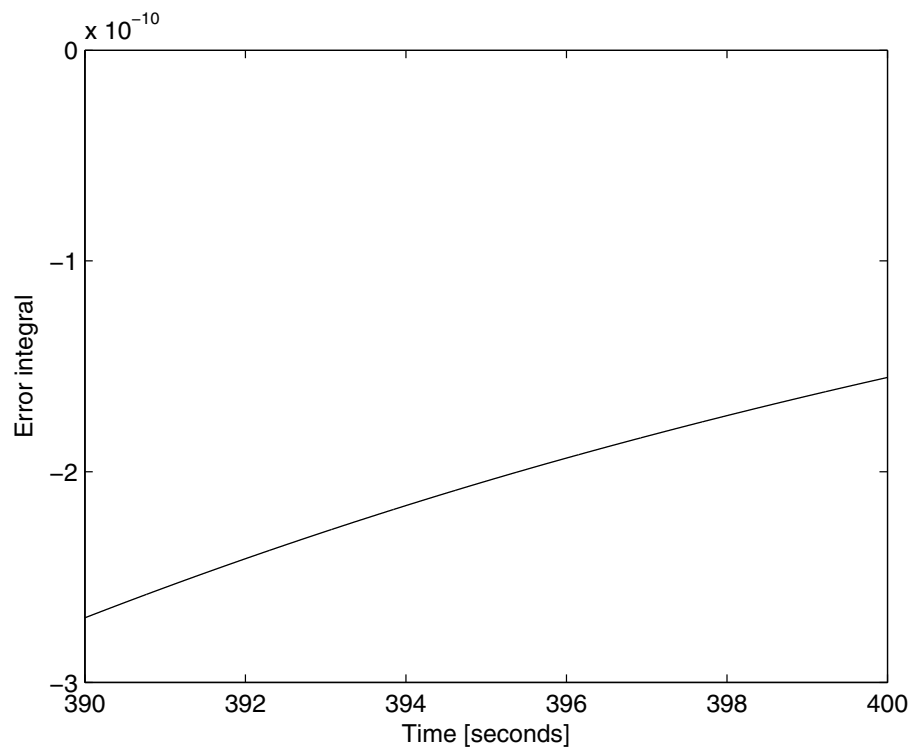

Fig. 10. Controller Performance: Error Integral (detail).

\section{Conclusions}

An extension to the traditional PID controller has been presented that incorporates an adaptive gain. The adaptive gain PID controller presented is demonstrated to asymptotically stabilize the system, this is shown in the simulations where the position error converges to zero.

In the presented analysis, considerations using known bounds of the system (such as friction coefficients) are used to show the stability of the system as well as to tune the controller gains $K_{p}$ and $K_{d}$.

\section{References}

Alvarez, J.; Santibañez, V. \& Campa, R. (2008). Stability of Robot Manipulators Under Saturated PID Compensation. IEEE Transactions on Control Systems Technology, Vol. 16, No. 6, Nov 2008, 1333 - 1341, ISSN 1063-6536

Ang, K. H.,; Chong, G. \& Li, Y. (2005). PID Control System Analysis, Design, and Technology. IEEE Transactions on Control Systems Technology, Vol. 13, No. 4, Jul 2005, 559 - 576, ISSN 1063-6536

Canudas de Wit, C. ; Olsson, H. ; Astrom, K.J. \& Lischinsky, P. (1995). A new model for control of systems with friction. IEEE Transactions on Automatic Control, Vol. 40, No. 3, Mar 1995, $419-425$, ISSN 0018-9286

Chang, P. H. \& Jung J.H. (2009). A Systematic Method for Gain Selection of Robust PID Control for Nonlinear Plants of Second-Order Controller Canonical Form. IEEE Transactions on Automatic Control, Vol. 17, No. 2, Mar 2009, 473 - 483, ISSN 1063-6536 
Distefano, J. J.; Stuberud, A. R \& Williams, I. J.(1990). Feedback and Control Systems, 2nd Edition, McGraw Hill, ISBN: 0-13228024-8, Upper Saddle River, New Jersey.

Guerra, R.; Acho, L. \& Aguilar L.(2005). Chattering Attenuation Using Linear-in-the-Parameter Neural Nets in Variable Structure Control of Robot Manipulators with friction. Proceedings of the International Conference on Fuzzy Systems and Genetic Algorithms 2005, pp. 65 - 75, Tijuana, Mexico, October 2005.

Hench, J. J. (1999). On a class of adaptive suboptimal Riccati-based controllers. Proceedings of the: American Control Conference, 1999., pp. 53 - 55, ISBN: 0-7803-4990-3, San Diego, CA, June 1999.

Kelly, R.; Santibáñez, V. \& Loría, A. (1996). Control of Robot Manipulators in Joint Space, Springer, ISBN: 978-1-85233-994-4, Germany.

Makkar, C.; Dixon, W.E.; Sawyer, W.G. \& Hu, G. (2005). A new continuously differentiable friction model for control systems design. Proceedings of the 2005 IEEE/ASME International Conference on Advanced Intelligent Mechatronics, pp. 600 - 605, ISBN: 0-7803-9047-4, Monterey, CA, July 2005.

Su, Y.; Müller P. C. \& Zheng, C. (2010). Global Asymptotic Saturated PID Control for Robot Manipulators. IEEE Transactions on Control Systems Technology, Vol. 18, No. 6, Nov 2010, 1280 - 1288, ISSN 1063-6536

Zhang, T. \& Ge, S. S. (2009). Adaptive Neural Network Tracking Control of MIMO Nonlinear Systems With Unknown Dead Zones and Control Directions. IEEE Transactions on Neural Networks, Vol. 20, No.3, Mar 2009, 483 - 497, ISBN 1045-9227 


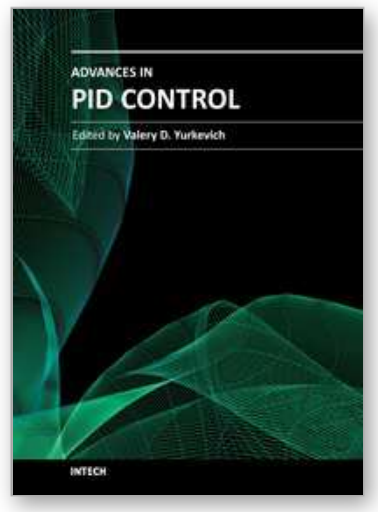

\author{
Advances in PID Control \\ Edited by Dr. Valery D. Yurkevich
}

ISBN 978-953-307-267-8

Hard cover, 274 pages

Publisher InTech

Published online 06, September, 2011

Published in print edition September, 2011

Since the foundation and up to the current state-of-the-art in control engineering, the problems of PID control steadily attract great attention of numerous researchers and remain inexhaustible source of new ideas for process of control system design and industrial applications. PID control effectiveness is usually caused by the nature of dynamical processes, conditioned that the majority of the industrial dynamical processes are well described by simple dynamic model of the first or second order. The efficacy of PID controllers vastly falls in case of complicated dynamics, nonlinearities, and varying parameters of the plant. This gives a pulse to further researches in the field of PID control. Consequently, the problems of advanced PID control system design methodologies, rules of adaptive PID control, self-tuning procedures, and particularly robustness and transient performance for nonlinear systems, still remain as the areas of the lively interests for many scientists and researchers at the present time. The recent research results presented in this book provide new ideas for improved performance of PID control applications.

\title{
How to reference
}

In order to correctly reference this scholarly work, feel free to copy and paste the following:

Ricardo Guerra, Salvador González and Roberto Reyes (2011). Adaptive Gain PID Control for Mechanical Systems, Advances in PID Control, Dr. Valery D. Yurkevich (Ed.), ISBN: 978-953-307-267-8, InTech, Available from: http://www.intechopen.com/books/advances-in-pid-control/adaptive-gain-pid-control-for-mechanicalsystems

\section{INTECH}

open science | open minds

\section{InTech Europe}

University Campus STeP Ri

Slavka Krautzeka 83/A

51000 Rijeka, Croatia

Phone: +385 (51) 770447

Fax: +385 (51) 686166

www.intechopen.com

\section{InTech China}

Unit 405, Office Block, Hotel Equatorial Shanghai

No.65, Yan An Road (West), Shanghai, 200040, China

中国上海市延安西路65号上海国际贵都大饭店办公楼 405 单元

Phone: +86-21-62489820

Fax: $+86-21-62489821$ 
(C) 2011 The Author(s). Licensee IntechOpen. This chapter is distributed under the terms of the Creative Commons Attribution-NonCommercialShareAlike-3.0 License, which permits use, distribution and reproduction for non-commercial purposes, provided the original is properly cited and derivative works building on this content are distributed under the same license. 